\title{
COMUNICAÇÃO CIENTÍFICA \\ Nota sobre a biologia de Cinomops abrasus (Temminck) (Mammalia, Chiroptera, Molossidae) no Rio de Janeiro, Brasil
}

\author{
Carlos E. L. Esbérard \& Helena G. Bergallo \\ Departamento de Ecologia, Universidade do Estado do Rio de Janeiro. Rua São Francisco Xavier 524, 20559-900 Rio de \\ Janeiro, Rio de Janeiro, Brasil. E-mail: cesberard@terra.com.br
}

\begin{abstract}
Notes on the biology of the Cinomops abrasus (Temminck), Rio de Janeiro, Brazil (Mammalia, Chiroptera, Molossidae). Data on Cinomops abrasus (Temminck, 1826) biology are presented, from 36 animals captured in Rio de Janeiro State, southeastern Brazil, between 1993 and 1997, from four different localities. KEY WORDS. Activity, occurrence, reproduction, roost.
\end{abstract}

RESUMO. São aqui apresentados dados sobre a biologia de Cinomops abrasus (Temminck, 1826) com base em 36 exemplares capturados no Estado do Rio de Janeiro, sudeste do Brasil, entre 1993 e 1997, em quatro diferentes localidades.

PALAVRAS CHAVE. Atividade, ocorrência, refúgio, reprodução.

A espécie Cinomops abrasus (Temminck, 1826) ocorre da Colômbia, Venezuela, Guiana, Peru, Brasil, Paraguai e norte da Argentina (Koopman 1993). A biologia de C. abrasus é pouco conhecida, pois é uma espécie pouco citada em inventários e em estudos ecológicos. Por meio de elevado esforço de coleta realizado entre 1989 e 2002, no Estado do Rio de Janeiro, totalizando 700 noites, 57 diferentes localidades e mais de 18000 capturas, foram obtidos 36 exemplares desta espécie, de quatro localidades. Este trabalho apresentada aspectos inéditos da biologia deste morcego.

O material examinado foi coletado em dois municípios do Rio de Janeiro: (1) Rio de Janeiro (residência no Bairro de São Cristóvão), um macho em 04/XI/1997; (Parque da Quinta da Boa Vista, 22 $54^{\prime} 0,6^{\prime \prime} \mathrm{S}$ e $43^{\circ} 13^{\prime} 25,8^{\prime \prime} \mathrm{W}, 45 \mathrm{~m}$ de altitude), um macho e 13 fêmeas em 30/V/1996; (Parque da Gávea, $23^{\circ} 58^{\prime} 68,7^{\prime \prime}$ S e $043^{\circ} 14^{\prime} 54,3^{\prime \prime} \mathrm{W}, 450 \mathrm{~m}$ de altitude), duas fêmeas em 01/VII/1993, cinco fêmeas em 24/VIII/1993, um macho e quatro fêmeas em 09/X/1994; um macho e três fêmeas em 06/I/1995, um macho em 15/VI/1997, uma fêmea em 20/IV/ 1998 e dois machos em 22/X/1998; (2) Petrópolis (Reserva Biológica de Araras, $22^{\circ} 31^{\prime} 6,2^{\prime \prime} \mathrm{S}$ e $43^{\circ} 10^{\prime} 0,12^{\prime \prime} \mathrm{W}, 1100 \mathrm{~m}$ de altitude), dois machos em 08/I/1994. Material testemunho da espécie está depositado na coleção de referência do Projeto Morcegos Urbanos (IBAMA, Processo número 1755/89).

Até o momento, o único registro publicado sobre este morcego no Estado do Rio de Janeiro, é de Ávila-PIrEs \& GouveIA (1977), que capturaram (entre 1954 e 1960) dois machos e três fêmeas a 800-850 m de altitude, no Parque Nacional de Itatiaia, Rio de Janeiro. PAtterson et al. (1996) capturaram esta espécie no Peru a 600 m de altitude. A ocorrência desta espécie na Reserva Biológica de Araras eleva a 1100 m o máximo de altitude já registrado para a espécie.

Em uma das capturas efetuadas no interior de residência, foi observado um exemplar adulto, refugiado em sótão, coabitando com Molossus molossus (Pallas, 1766), no bairro de São Cristóvão, município do Rio de Janeiro (Fig. 1). Outro refúgio desta espécie foi descoberto em um exemplar de árvore "Sumaúma" (Pseudobombax marginatum (A. St-Hil, A. Juss \& Cambess.), Bombacaceae). Este abrigo apresentava abertura situada à cerca de $6 \mathrm{~m}$ de altura do solo, com $30 \mathrm{~cm}$ de largura e $15 \mathrm{~cm}$ de altura. O oco estendia-se por cerca de 1,30 m, com diâmetro máximo de $35 \mathrm{~cm}$. Neste abrigo capturamos, após o corte do tronco com motoserra para a manutenção da rede elétrica, um macho com testículos escrotados e 13 fêmeas inativas.

O uso de refúgio diurno em ocos de árvores é um padrão conhecido para espécies de Molossidae (e.g. TAdDei \& Vizotto 1976, TADDEI et al. 1976). O uso de residência para refúgio pode indicar que esta espécie pode se adaptar ao meio urbano, como já sugerido por TADDEI et al. (1976) e já observado para Molossus molossus (Pallas, 1766) e Molossus rufus (MARques 1986, Esbérard 2002).

Vinte e um exemplares foram capturados com redes de neblina armadas sobre dois lagos artificiais (Reserva Biológica de Araras, Petrópolis e Parque da Gávea, Rio de Janeiro). Na primeira localidade, capturamos também Molossus molossus que se refugiava em telhado distante cerca de $50 \mathrm{~m}$, onde não encontramos C. abrasus. No Parque da Gávea uma grande diversidade de morcegos insetívoros foi coletada, incluindo Myotis 


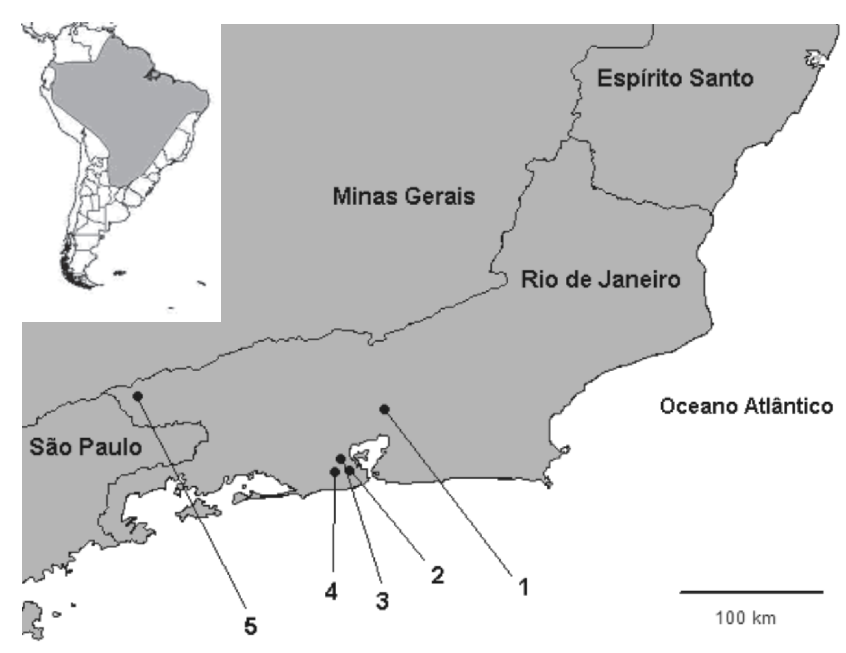

Figura 1. Localidades onde Cinomops abrasus for capturauu ıu Estado do Rio de Janeiro e distribuição geográfica da espécie, segundo EISENBERG \& REDFORD (1999). (1) Reserva Biológica de Araras, Petrópolis; (2) residência, Rio de Janeiro; (3) Parque da Quinta da Boa Vista, Rio de Janeiro; (4) Parque da Cidade, Rio de Janeiro; (5) Parque Nacional de Itatiaia, Itatiaia (ÁVILA-PIREs \& GouvEIA, 1977).

nigricans (Schinz, 1821), Lasiurus ega (Gervais, 1856), Eptesicus brasiliensis (Desmarest, 1819) (Vespertilionidae), Molossus molossus, Molossus rufus (E. Geoffroy, 1805), Eumops auripendulus (Schaw, 1800), Nictynomops macrotis (Gray, 1840) e Tadarida brasiliensis (I. Geoffroy, 1824) (Molossidae) (EsbéRARD 2003). Amostras fecais de seis exemplares de C. abrasus foram analisadas e continham fragmentos de insetos e algas verdes.

Quatro fêmeas foram capturadas com feto palpável no mês de janeiro e três machos apresentaram testículos escrotados, um em junho e dois em agosto. Não foi observada diferença entre os sexos quanto ao comprimento de antebraço (machos, $\mathrm{N}=8$, média de $41,11 \pm 1,82 \mathrm{~mm}$; fêmeas, $\mathrm{n}=25$, média de $41,01 \pm 1,11 \mathrm{~mm})(\mathrm{t}=0,165, \mathrm{p}=0,873)$ e peso (machos, $\mathrm{N}=$ 8 , média de $24,13 \pm 4,54 \mathrm{~g}$; fêmeas, $\mathrm{N}=25$, média de $24,40 \pm 2,74 \mathrm{~g})(\mathrm{t}=0,606, \mathrm{p}=0,696)$.

Cinomops abrasus apresentou reprodução estacional, com fêmeas capturadas grávidas no verão. A maior parte dos Molossídeos tropicais são poliestrais, variando o número de ciclos reprodutivos em diferentes localidades (KRUTZCH 2000). A espermatogênese pode ser contínua ou presente na maior parte do ano, servindo a fêmeas poliestricas, como demonstrado por KRutzch \& Chichton $(1985,1990)$. A captura de fêmeas com fetos palpáveis em janeiro sugere que este morcego apresente a mesma estacionalidade que a observada em espécies dos gêneros Molossops e Molossus para o sudeste do Brasil, que estão ativas sexualmente na primavera e verão (e.g. TADDEI et al. 1976, TADdei \& VizotTo 1976, EsbéraRd 2002). Devido ao reduzido número de observações não é possível afirmar se esta espécie apresenta um ou dois partos a cada época reprodutiva.
A espécie C. abrasus apresentou atividade durante toda a noite, sendo observados dois picos de capturas. O primeiro picom ocorreu logo após o pôr-do-sol (30 a 60 minutos após o pôr-do-sol) e o outro imediatamente antes do amanhecer (600 a 690 minutos após o pôr-do-sol) (Fig. 2).

A captura desta espécie sobre a água e a presença de algas nas fezes demonstram o uso de coleções de água para a ingestão e/ou captura de insetos.

Como Cinomops planirostris (Peters, 1865), parece que $C$. abrasus forma haréns, diferindo do observado para Molossops temminckii (Burmeister, 1854), que foi observado isolado ou em casais (TADDEI \& VizotTo 1976).

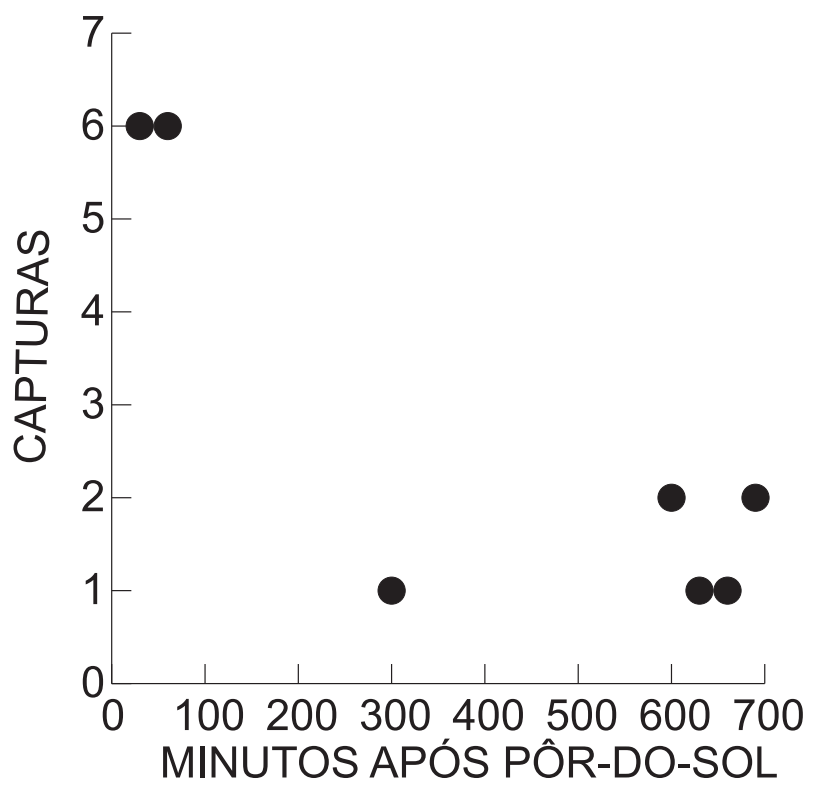

Figura 2. Horário de captura em redes de neblina armadas sobre coleções de água, agrupadas a intervalos de 30 minutos após o pôr-do-sol, usando as efemérides astronômicas (Observatório Nacional 1993-1997).

\section{AGRADECIMENTOS}

Agradecemos ao Dr. Renato Gregorin à confirmação e o fornecimento de informações sobre a espécie e a Dra Ludmilla Aguiar e Dr. Nélio Roberto dos Reis as críticas.

\section{REFERÊNCIAS BIBLIOGRÁFICAS}

Ávila-Pires, F.D. \& E. Gouveia. 1977. Mamíferos do Parque Nacional de Itatiaia. Boletim do Museu Nacional, nova série, Rio de Janeiro, 291: 1-29.

EsbérARD, C.E.L. 2002. Composição da colônia e reprodução de Molossus rufus em refúgio no sudeste do Brasil (Mammalia, Molossidae). Revista Brasileira de Zoologia, Curitiba, 19

Revista Brasileira de Zoologia 22 (2): 514-516, junho 2005 
(4): 1153-1160.

EsbÉRARD, C.E.L. 2003. Diversidade de morcegos em uma área de Mata Atlântica regenerada no sudeste do Brasil (Mammalia: Chiroptera). Revista Brasileira de Zoociências, Juiz de Fora, 35 (2): 189-204.

EISENBERG, J.F.\& K.H. REDFORD. 1999. Mammals of the neotropics. The Northern Neotropics. The Central Neotropics: Ecuador, Peru, Bolivia, Brazil. Chicago, University of Chicago Press, vol. 3, 609p.

Koopman, K.F. 1993. Order Chiroptera, p. 137-241. D.E. Wilson \& D.M. ReEDer (Eds). In: Mammal species of the world, a taxonomic and geographic reference. Washington, Smithsonian Institution Press, 1206p.

Krutzsch, P.H. 2000. Anatomy, phisiology and ciclicity of the male reproductive tract, p. 91-155. In: E.G. CRICHTON \& P.H. KRUTZSCH (Eds). Reproductive biology of bats. Cambridge, Academic Press, Cambridge, 510p.

Krutzsch, P.H. \& E.G. Chichton. 1985. Observations on the reproductive cycle of female Molossus fortis (Chiroptera:
Molossidae) in Puerto Rico. Journal of Zoology, London, 207(A): 137-150.

Krutzsch, P.H. \& E.G. Chichton. 1990. Observations on the reproductive anatomy of the male Molossus fortis (Chiroptera: Molossidae) with comments on the chronology of the reproductive events. Mammalia, Paris, 54: 287-296.

MARQUes, S.A. 1986. Activity cicle, feeding and reproduction of Molossus ater (Chiroptera: Molossidae) in Brazil. Boletim do Museu Paraense Emilio Goeldi, Belém, 2: 159-179.

Patterson, B.D.; V. Pacheco \& S. Solari. 1996. Distribution of bats along an elevational gradient in the Andes of southeastern Peru. Journal of Zoology, London, 240: 637-658.

Taddei, V.A.; L.D. Vizotto. 1976. Notas sobre Molossops temminckii temminckii e Molossops planirostris (Chiroptera Molossidae). Naturalia, São Paulo, 2: 47-59.

TAdDeI, V.A.; L.D. Vizotto \& S.M. Martins. 1976. Notas taxionômicas e biológicas sobre Molossops brachymeles cerastes (Thomas, 1901) (Chiroptera - Molossidae). Naturalia, São Paulo, 2: 61-69.

Recebido em 05.VIII.2004; aceito em 26.V.2005. 